\title{
Knickung gerader Stäbe durch Druck und konservative Torsion
}

\section{Doctoral Thesis}

Author(s):

Beck, Max

Publication date:

1955

Permanent link:

https://doi.org/10.3929/ethz-a-000099286

Rights / license:

In Copyright - Non-Commercial Use Permitted 


\title{
KNICKUNG GERADER STÄBE DURCH \\ DRUCK UND KONSERVATIVE TORSION
}

\author{
VON DER \\ EIDGENÖSSISCHEN TECHNISCHEN \\ HOCHSCHULE IN ZÜRICH
}

ZUR ERLANGUNG

DER WÜRDE EINES DOKTORS DER

TECHNISCHEN WISSENSCHAFTEN

\section{GENEHMIGTE \\ PROMOTIONSARBEIT}

VORGELEGT VON

MAX BECK

VON SCHAFFHAUSEN

REFERENT: HERR PROF. DR. H. ZIEGLER KORREFERFNT: HERR PROF. DR. E. STIEFEL

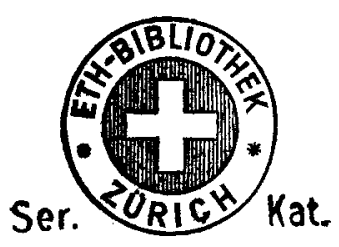

1955

SPRINGER - VERLA G BERLIN W 35 


\section{Knickung gerader Stäbe durch Druck und konservative Torsion*.}

\section{Von M. Beck.}

1. Einleitung. L. Euler hat im Jahre 1757 als Erster die axiale Druckkraft berechnet ${ }^{1}$, unter der ein schlanker Stab ausknickt. Ein seitliches Ausbiegen kann auch bei einem Stab auftreten, der ein Drehmoment zu übertragen hat. Die kritische Belastung eines gedrückten und tordierten Stabes hat für kreisförmigen Querschnitt zuerst A. G. Greenhill ${ }^{2}$ untersucht, während $H$. Grammel ${ }^{3}$ für die Lagerung V von Abb. 4 eine Lösung für beliebige Querschnitte berechnet hat. Diese Arbeiten beruhen auf der Annahme, daß der Momentvektor beim Ausknicken seine ursprüngliche axiale Richtung beibehalte. Das merkwürdige Ergebnis, daß bei den Lagerungen III und IV in Abb. 4 ein beliebig kleines Moment den Stab zu knicken vermag, regte $H$. Ziegler ${ }^{4}$ zu einer genaueren t’berprüfung des belastenden Momentes an. Dabei zeigte sich zunächst, daß ein axiales Moment nichtkonservativ ist. Nichtkonservative Stahilitätsprobleme müssen aber mit dem kinetischen Stabilitätskriterium (Untersuchung der kleinen Schwingungen um die Gleichgewichtslage) behandelt werden, während das von Greenhill und Grammel verwendete einfachere statische (Bestimmung kritischer Belastungen durch Ermittlung der nichttrivialen Gleichgewichtslagen) und das energetische Kriterium (für die kritische Belastung ist die potentielle Energie erstmals nicht mehr positiv definit) nur bei konservativen Systemen verwendet werden dürfen ${ }^{5}$. Die von $A$. Trösch ${ }^{6}$ durchgeführten Schwingungsrechnungen ergeben, daß selbst bei Einführung von Dämpfungskräften die erwähnten unbefriedigenden Resultate nur zum Teil korrigiert werden.

Indem H. Ziegler ${ }^{7}$ auf die den Momentvektor erzeugenden Einzelkräfte zurückging, stellte er fest, daß sich für die praktisch wichtigsten Belastungsarten konservative Momente ergeben (vgl. auch Abschnitt 2 b), während Kräfteverteilungen, die axial bleibende Momente liefern, wohl ziemlich selten vorkommen. Mit den von ihm herausgegriffenen Belastungen definierte er das semi-, das quasi- und das pseudotangentiale Moment und zeigte, daß mit diesen Momenten beim Stab mit zwei gleichen Biegesteifigkeiten für alle fünf Arten der Lagerung in Abb. 4 plausible Knicklasten gefunden werden ${ }^{8,9}$.

Die folgende Arbeit stellt eine Erweiterung der Rechnungen von H. Ziegler dar. Unter allgemeinen Voraussetzungen werden im Abschnitt 2 die Ausgangsgleichungen abgeleitet. Eine Lösung ohne Einschränkungen führt indessen auf sehr unübersichtliche Knickgleichungen. Wir lassen daher im Abschnitt 3 einen Stab mit zwei verschiedenen Biegesteifigkeiten zu, der zwar sogar eine spannungsfreie Verwindung aufweisen kann, jedoch vor dem Knicken nur auf Torsion beansprucht sein soll. Im Abschnitt 4 wird dann bei einem Stab mit zwei gleichen Biegesteifigkeiten auch eine Druckkraft mitberücksichtigt. Insbesondere die Knickgleichungen dieses Abschnittes werden eingehend diskutiert und die kritischen Dynamen in Diagrammen wiedergegeben.

* Von der ETH in Zürich genehmigte Promotionsarbeit.

${ }^{1}$ L. Euler, Histoire de l'Académie, Bd. 13, Berlin 1757.

${ }^{2}$ A. G. Greenhill, Proc. Inst. Mech. Eng. 1883, S. 182.

3 R. Grammel, Z. angew. Math. Mech. 3, (1923), S. 262; siehe auch C. B. Biezeno u. R. Grammel, Technische Dynamik, Bd. 1, S. 607, 2. Aufl. 1953.

4 H. Ziegler, Z. angew. Math. Physik 2, (1951) S. 265. H. Ziegler stellte ferner fest, daß für verschiedene Arten der Lagerung jede Drehzahl kritisch ist, sobald die Welle ein beliebig kleines axiales Moment zu übertragen hat.

${ }^{5}$ H. Ziegler, Ing.-Arch. 20 (1952), S. 49 und H. Ziegler, Z. angew. Math. Physik 4 (1953), S. 90 und 168. Die erste Arbeit demonstriert das Versagen des statischen und des energetischen Stabilitätskriteriums bei einem einfachen nichtkonservativen Stabilitätsproblem, während die zweite eine grundzätzliche Analyse der linearen Stabilitätsprobleme und der Methoden zu ihrer Lösung darstellt.

6 A. Trösch, Ing.-Arch. 20 (1952), S. 258.

${ }^{7}$ H. Ziegler, Z. angew. Math. Physik 3 (1952), S. 96.

8 Auch die Berechnung des Einflusses der Torsion auf die kritischen Drehzahlen ergibt für diese drei Momente keine Überraschungen mehr. H. Ziegler, Ing.-Arch. 20 (1952), S. 377.

o Einen weiteren, mit dem pseudotangentialen Moment verwandten Fall, dje Übertragung des Momentes durch ein Kreuzgelenk, behandelt $W . T$. Koiter in einer noch nicht veröffentlichten Arbeit. 